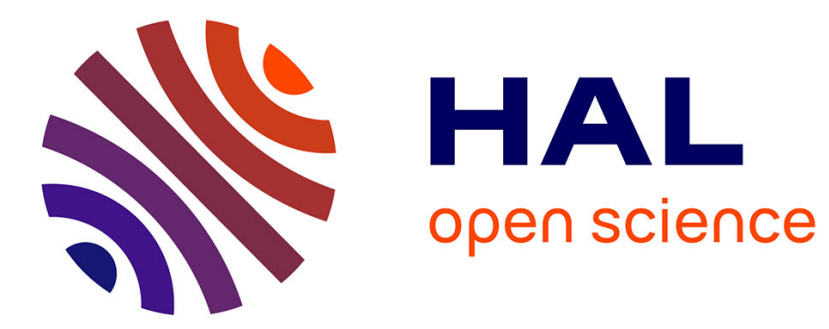

\title{
La logique peut-elle mouvoir l'esprit?
}

Pascal Engel

\section{To cite this version:}

Pascal Engel. La logique peut-elle mouvoir l'esprit?. Dialogue: Canadian Philosophical Review / Revue canadienne de philosophie, 1998, 37 (1), pp.35-53. halshs-01200559

\section{HAL Id: halshs-01200559 \\ https://shs.hal.science/halshs-01200559}

Submitted on 24 Sep 2015

HAL is a multi-disciplinary open access archive for the deposit and dissemination of scientific research documents, whether they are published or not. The documents may come from teaching and research institutions in France or abroad, or from public or private research centers.
L'archive ouverte pluridisciplinaire HAL, est destinée au dépôt et à la diffusion de documents scientifiques de niveau recherche, publiés ou non, émanant des établissements d'enseignement et de recherche français ou étrangers, des laboratoires publics ou privés. 
LA LOGIQUE PEUT-ELLE MOUVOIR L'ESPRIT?

\author{
Pascal Engel \\ Paris IV
}

Exposé au séminaire d'épistémologie comparative

Aix en Provence 1997

Dialogue, 1998, I, 1-27

Un siècle après leur dialogue narré par Lewis Carroll, Achille et la Tortue se retrouvèrent au bout du chemin, sur la ligne d'arrivée. Le fier guerrier, dont la colère était retombée, avait repris du poil de la bête — si l'on peut dire cela d'un demi-dieu— en constatant qu'une fois de plus il avait gagné.

— “Tu vois, dit Achille, finalement j’ai encore réussi à prendre les devants, exactement comme il y a presque trois millénaires de cela. La distance qu'avait mise entre nous le cruel Zénon avait beau augmenter constamment, je suis quand même parvenu à te rejoindre et à te dépasser. Et quand Lewis Carroll a mis entre nous toute la distance qu'il y a entre accepter les prémisses d'une inférence et adopter sa conclusion, tu as quand même été obligée de tirer la conclusion. Comme je le disais il y un siècle, la logique " t'a prise à la gorge", et tu as dû quand même inférer $q$ à partir du moment où tu avais admis que $p$ et sip alors $q$ étaient vrais! Une fois encore, la logique a gagné. Elle ne peut pas perdre. C'est d'ailleurs ce qui fait d'elle la logique. Ses déductions sont sûres, l'une des caractéristiques de ses inférences est qu'elles sont monotones, c'est-à-dire que si A implique B, alors 
A et $\mathrm{C}$ impliquent aussi $\mathrm{B}$. Ce n'est pas le cas pour les inférences inductives, où il suffit parfois d'ajouter ou de retirer une prémisse pour que la conclusion ne s'ensuive plus. Et nous savons comment justifier nos principes de raisonnement pour la logique déductive classique: ses règles d’inférences ne sont pas simplement non contradictoires, elles sont aussi correctes et complètes. Un sage aixois ${ }^{1}$ a même dit que l'on avait affaire, quand il s'agit de la logique propositionnelle, au degré zéro du contenu formel, où tout tourne correctement parce que les objets et les opérations coincident parfaitement. Toute roule bien, et pierre qui roule n'amasse pas mousse.

— Minute, répondit la Tortue, je ne vois pas les choses ainsi. Tu crois avoir gagné, et pouvoir te reposer tranquillement sur mon dos, mais je pense que tu rêves tout éveillé, fier guerrier. Tu as passé trop de temps sous ta tente, et cela te trouble l'esprit. Je me permets de te rappeler où nous en étions la fois précédente. Tu me demandais, n'est-ce pas, de considérer les deux propositions d'Euclide:

(A) Des choses qui sont égales à la même chose sont égales entre elles.

(B) Les deux côtés de ce triangle sont des choses qui sont égales à la même chose.

et de reconnaittre que quiconque accepte (A) et (B) doit accepter la proposition suivante:

(Z) Les deux côtés de ce triangle sont égaux entre eux.

— Et je t'ai fait remarquer alors, rappela Achille, que n'importe quel individu sensé, et doué seulement des pouvoirs les plus minimaux de la raison, ne peut faire

\footnotetext{
${ }^{1}$ Gilles Granger, voir son livre Granger 1992
} 
autrement qu'accepter (Z). Mais toi, rêtif animal, tu as refusé de l'accepter. Je t’ai alors présenté la proposition suivante:

(C) Si A et B sont vrais, alors ( $Z$ ) doit être vrai

— Et je l'ai acceptée, dit la Tortue. Mais je te faisais remarquer que comme (C) est une proposition distincte de $(A)$ et de $(B)$ je pouvais très bien accepter $(C)$ sans accepter (A) et (B). En retour, je pouvais très bien accepter (A) et (B) sans accepter $(\mathrm{C})$. Et dans un cas comme dans l'autre, rien ne me forçe à accepter $(\mathrm{Z})$. Tu m'as alors proposé une autre proposition:

(D) $\mathrm{Si} \mathrm{A}$ et $\mathrm{B}$ et $\mathrm{C}$ sont vrais, alors $\mathrm{Z}$ doit être vrai.

Et comme (D) est une proposition distincte de (A), (B) et (C), je peux l'accepter sans accepter l'une ou l'autre de ces propositions, ou sans les accepter toutes les trois. Et l'affaire peut continuer à l'infini. Je n'ai pas accepté (Z). Et les choses en sont restées là. Je ne vois toujours pas pourquoi tu considères avoir gagné cette course à l'inférence. Puis-je te rappeler ce que Lewis Carroll écrivit quand George Stout, l'éditeur de Mind, lui demanda ce que voulait dire l'histoire?

"Mon paradoxe... repose sur le fait que, dans une Hypothétique, la vérité de la Protase, la Vérité de l'Apodose, et la validité de la suite, sont trois propositions distinctes. Par exemple, si j'admets

(A) Tous les hommes sont mortels

et

(B) Socrate est un homme 
(C) La suite "Si tous les hommes sont mortels, et si Socrate est un homme, alors

$$
\text { Socrate est morte" est valide }
$$

alors je n'admets pas

(Z) Socrate est mortel.

A nouveau, si j'admets $(\mathrm{C})$ mais pas $\mathrm{A}$ et $\mathrm{B}$, je ne parviens toujours pas à admettre Z. Par conséquent, avant d'admettre Z, je dois admettre A et B et C. [A présent considérez]

(D) Si A et B et $\mathrm{C}$ sont vrais, alors $\mathrm{Z}$ est vrai.

A présent supposez que je nie que cette dernière suite soit valide. Supposez que je dise "J'admets A et B mais je n'admets pas que je sois par là obligé d'admettre Z". Il ne fait pas doute que je doisattendre, avant d'admettre Z, que l'on m'ait fait voir la validité de cette suite: c'est-à-dire que pour admettre $Z$, je dois admettre $A, B, C$ et $\mathrm{D}$ ! et Ainsi de suite. Je pense que vous découvrirez que cela continue comme dans "La maison que Jacques a bâtie". (C.L. Dogson 1977, p.472, cité par Smiley 1995 p.725-6)

— J'entends bien, répondit Achille, agacé qu'on lui rappelle cet épisode humiliant. Mais tu fais comme si les logiciens avaient purement et simplement accepté la régression à l'infini de notre maître Lewis Carroll. Mais ils ont depuis longtemps diagnostiqué ce qui n'allait pas dans l'histoire dont nous étions jadis les infortunés protagonistes. Le problème, ont-ils noté, est que Lewis Carroll (mais je le confesse, aussi nous-mêmes par la même occasion) ne faisions pas la distinction 
entre une proposition vraie de la logique et une règle d'inférence. A partir du moment où l'on reconnaît que le modus ponens est une règle d'inférence (quelquefois appelée de détachement) correcte de la logique classique, il n'y a aucun besoin de la considérer comme une proposition vraie que l'on aurait besoin d'ajouter au raisonnement, comme on le ferait pour une prémisse supprimée dans un enthymème, pour pouvoir tirer validement la conclusion. Voici ce que dit par exemple le logicien Timothy Smiley dans un article récent sur le paradoxe de Lewis Carroll :

"Le coeur du paradoxe est l'étape qui introduit C, et dont l'itération introduit D, etc. Si j'admets $A$ et $B$ mais pas $C$, je ne parviens pas à admettre $Z$. A nouveau, si j'admets $C$, mais que je n'admets pas $A$ et $B$, je ne parviens toujours pas à admettre Z. Par conséquent, avant d'admettre $Z$, je dois admettre $A, B$ et $C$. Et la solution à ce point est viciée par une ambiguité. Si “je n’admets pas C”, cela doit être que j’ai envisagé C mais que je ne suis pas parvenu à l'accepter, ou même que je l'ai nié. Ou c'est peut-être parce que je n'ai jamais envisagé $C$, ce qui, naturellement, a fait que je n'ai jamais eu l'occasion de l'admettre. Dans le premier cas la prémisse de Carroll “Si j’admets A et B mais pas C, je ne parviens pas à admettre Z” est certainement vraie, mais tout ce qui s'ensuit est au plus ceci: "Avant d'admettre Z, je dois admettre $\mathrm{A}$ et $\mathrm{B}$ et soit admettre $\mathrm{C}$ ou l'avoir l'avoir considéré." Aucune régression ne menace ici. Dans le second cas, la prémisse est fausse. C'est un fait concernant l'inférence que je puisse — ce que les gens font raisonablement et légitimementinférer Z, et ressentir toutes les obligations qui s'ensuivent, sans jamais envisager C. Avant d'admettre $Z$, tout ce que j'ai besoin de faire c'est admettre $A$ et $C$ et inférer Z. A nouveau aucune régression à l'infini."

(Smiley 1995, p.726)

C'est le simple bon sens. Geach avait fait le même diagnostic dans "Assertion" (Geach 1965) quand il critiquait la conception des "tickets d’inférence" de Ryle. 
Dans "If, so and Because", Ryle (1954) voyait correctement que dans " $\mathrm{Si} p$ alors $q$; donc $q$ " l'hypothétique n'est pas une prémisse coordonnée à “ $p$ ". Mais il soutenait que c'est une "licence" ou une autorisation à effectuer l'inférence " $p$ donc $q$ " quand on a la prémisse " $p$ ". Contre la conception traditionnelle du modus ponens il soutenait que si nous avions besoin de fournir "si $p$ alors $q$ " comme une prémisse pour l'inférence de " $q$ " à partir de " $p$ " et "si $p$ alors $q$ " nous nous embarquerions précisément dans la régression à l'infini de Lewis Carroll. Mais Geach n'admet pas cet argument de Ryle:

"Des lectures particulières de " $p$ " et de " $q$ " faire de " $p$, donc $q$ " un argument logiquement valide; mais ce n'est pas en général logiquement valide, et si cela ne l'est pas, alors aucun pouvoir au ciel ou sur terre ne peut me fournir une "licence" que le rende logiquement valide. D'un autre côté, "si $p$ alors $q$, mais $p$, donc $q$ " est logiquement valide; et cela veut dire précisément que les deux prémisses "si $p$ alors $q$ " et " $p$ "sont suffisantes pour produire la conclusion " $q$ ". Il n'y a donc pas lieu d'introduire une prémisse supplémentaire, et la régression ne démarre jamais." (Geach 1965, in Geach 1972, p. 258-9)

Le raisonnement de Geach est également sous-tendu par ce qu'il appelle "the Frege point": il faut distinguer une proposition qui a une occurence comme assertée (et devant laquelle on place le signe d'assertion ou la barre de jugement frégéenne), comme " $p$ “ dans la raisonnement en modus ponens, de cette proposition comme non assertée dans " si $p$ alors $q$ " dans le même raisonnement. Si nous voulons comprendre le modus ponens, il nous faut le comprendre ainsi :
(i) $\mathrm{I}-\mathrm{p}$
(ii) Íx si $\mathrm{p}$ alors $\mathrm{q}$
(iii) $\mathrm{I}-\mathrm{q}$ 
Le fait de voir que " $p$ " dans (i) est asserté alors qu'il n'est pas asserté dans (ii) mais la proposition complexe "si $p$ alors $q$ " — suffit à montrer la distinction. Car comme le dit Dummett, si ce n'était pas le cas, le modus ponens serait invalide, car alors

"Pierre est juif; si Pierre est juif, André est juif; par conséquent André est juif" serait le même que "Si à la fois Pierre est juif et si Pierre est juif André est juif, alors André est juif”, et le fait qu'il ne le sont pas était précisément la découverte de Lewis Carrol (dans "Ce que la Tortue dit à Achille)" (Dummett 1973, p.304)

Tout ces logiciens, chère Tortue, ont remis notre histoire sur pieds. Et la morale, c'est que tu dois bien inférer (Z) de (A) et de (B), comme je l'ai toujours dit. Et voilà pourquoi je peux, un siècle après notre premier échange, m’asseoir à nouveau confortablement sur ton dos, et savourer ma victoire.

— Eh bien non, répondit la Tortue, je ne m’admets pas vaincue pour autant. Je veux bien que le modus ponens soit une règle d’inférence valide de la logique déductive (même si certains logiciens ont soutenu que ce n'était pas le cas; voir Mc Gee 1985; mais je laisserai ici ce point de côté 2 ). Mais quand bien même elle l'est,

\footnotetext{
${ }^{2}$ Philippe Minh m'a fait remarquer que le raisonnement (A)-(B)-(Z) ci-dessus n'est pas simplement une instance du modus ponens, mais emploie aussi la règle d'instanciation universelle, puisqu'on passe d'un énoncé conditionnel quantifié universellement à un énoncé singulier pour aller de (A) à (B) (c'est encore plus clair dans l'exemple pris par Carroll dans sa lettre à Stout). Mais cela ne change rien au point avancé par Carroll. Achille peut montrer à la Tortue la validité de la règle d'instanciation universelle, et celle-ci peut l'accepter comme une prémisse hypothétique de son inférence, au même titre que la prémisse (C). La régression s'enclenchera aussi. Bref le Carroll point s'applique à toute règle de logique, et pas simplement au modus ponens.

La même remarque s'applique aussi à une observation que m'a faite Eric Audureau: si l'on admet, comme Achille le fait ci-dessus, que le modus ponens est une règle d'inférence de la logique élémentaire, et qu'on reformule celle-ci, dans la lignée de Gentzen, en termes de régles d'inférences, et non plus en termes d'axiomes ou de "lois du vrai" à la manière de Frege, ce que le paradoxe de Carroll montre n'est-il pas que l'on doit renoncer à parler de vérité en logique, et parler plutôt en termes de règles d'inférence, autrement dit adopter délibéremment une approche en termes syntactiques ou de théorie de la démonstration et non plus une approche seulement sémantique (sur ce contraste, voir Engel 1989)? L'erreur d'Achille, dans le dialogue initial, serait alors de demander à la Tortue de reconnaître que (C) est une proposition vraie. Mais ici aussi, je ne crois pas que le point de Carroll soit résolu quand on passe d'une conception sémantique de la logique à une conception syntactique ou en termes de déduction
} 
ou le serait, elle reste une règle qui est écrite dans les manuels de logique ordinaires. Toute règle qu'elle est, elle ne peut pas me forcer, en elle-même à accepter, ou à reconnaître que la conclusion $q$ s'ensuit des prémisses $p$ et sipalors $q$ - Car accepter ou reconnaittre une proposition comme vraie, ou inférer la conclusion des prémisses, sont des actes psychologiques. Et je ne vois pas en quoi le fait que les livres de logique me disent je dois inférer $q$ de $p$ et si $p$ alors $q$ pourrait me forcer à l'inférer. Je peux très bien ne pas croire que si une proposition A est vraie, alors une autre proposition B est vraie aussi. Je peux aussi croire que A est vraie et, pour une raison quelconque, ne pas croire que B est vrai ou s'ensuit. La logique en elle-même n'est qu'un ensemble de propositions écrites dans des manuels de logique. Elle ne nous dit rien des raisons que nous pouvons avoir de croire en la vérité de prémisses particulières, ou de croire en la vérité de conclusions qui s'ensuivent logiquement de ces prémisses. Même si nous appelons certaines propositions des règles, ces règles seront exprimées par des signes sur le papier, mais je ne vois pas pourquoi le fait qu'un manuel de logique les contienne pourrait me donner un quelconque motif pour faire les inférences correspondantes, à titre d'actes psychologiques. Et tu ne nies pas, n'est-ce pas, qu'il me faille entrer dans l'état mental d'inférer (Z) de (A) et de (B) pour pouvoir tirer cette conclusion. Bref comment la logique peut-elle mouvoir mon esprit, comment peut-elle par elle-même me mettre en mouvement? Et si elle ne le peut pas pour moi, pourquoi le pourrait-elle pour toi? Tu as beau être appelé "divin" par Homère, je ne crois même pas qu'un dieu, ni même un démon, aurait ce pouvoir de mouvoir mon esprit. (Stroud 1979, Engel 1989, p.373; Blackburn 1995 p.695)

L'erreur, Achille aux pieds légers, que tu fais, est la même que ceux qui croient que la logique a par elle-même un pouvoir normatif, le pouvoir qu'elle a par ses règles de nous enjoindre à effectuer des inférences - y compris des inférences que nous ne serions pas prêts à admettre intuitivement, contrairement au modus ponens. Je veux bien qu'avec les logiciens contemporains tu aies renoncé à la 
conception de la logique comme "hyperphysique" décrivant un univers de faits rigides, des "lois-de l'être vrai", comme aurait dit Frege, et que tu soutiennes avec eux que la logique est avant tout affaire de règles. Mais qu'on soutienne la conception "platonicienne" que défendait Frege ou qu'on admette, à la suite de Gentzen, Dummett, Prawitz, ou la plupart des contemporains, que la logique est mieux conçue comme un système de règles d'inférence "naturelles", il ne s'ensuit pas qu'on a expliqué comment elle peut être normative. En logique comme en morale, "doit" implique "peut". Mais tu ne m'as expliqué pourquoi il en est ainsi. C'est pourquoi je reste, comme on dit, froide devant tes arguments. Ils ne me font pas plus bouger que ne le faisait la régression à l'infini de Lewis Carroll. Ce n'est pas seulement par les pieds que tu es léger, ô héros!. C’est aussi par la tête.

— Mais alors, répondit Achille, ô stupide animal, où veux-tu aller chercher ce qui peut mouvoir ton esprit à tirer la conclusion d'une inférence aussi ridiculement simple, sinon dans ton esprit lui-même? Veux-tu me dire que c'est par une loi de ta psychologie que ton esprit pourra inférer (Z) de (A) et de (B), et qu'il n'y a que s'il existe une telle loi du mouvement de tes représentations de (A), (B) et (Z) (ou de tes associations neuronales correspondantes)? Admettons, ce qui est loin d'être évident, qu'il y ait une telle loi, juste à titre de supposition. Je veux bien qu'elle ait un pouvoir causal, tout comme les lois d'un système hydraulique représentent le pouvoir causal des mécanismes en question. Mais comment de telles lois causales auraient-elles un pouvoir normatif? Comment pourraient-elles me donner jamais une raison, comme tu le demandes, de tirer la conclusion? C'est comme si tu me disais que le courant qui passe à travers un fil électrique donne à l'ampoule de ma lampe une raison de s'allumer! Jamais une loi causale, encore moins une loi causale psychologique, n'a fait une norme. Et si la règle d'inférence du modus ponens te force à inférer la conclusion, c'est parce qu'elle est une norme du raisonnement 
correct. Et les normes du raisonnement correct sont ce qui nous oblige à tirer la conclusion. C’est pourtant simple.

— Je veux bien l'admettre, dit la Tortue. Laisse moi alors écrire sur mon calepin la proposition suivante:

(E) C'est une norme que si l'on accepte que (A) et (B) sont vraies, alors on devra accepter (Z).

Mais supposons que je n'accepte pas cette norme. Tu conviens alors que je ne pourrai pas inférer $(\mathrm{Z})$ ?

- Ce serait alors, dit Achille, pour reprendre le mot de Frege, "une nouvelle forme de folie"! Ce qui vaudrait pour nous comme norme pourrait ne pas valoir comme norme pour d'autres? Peut-être est-ce vrai des normes de la politesse ou de l'étiquette, mais comment cela pourrait-il être vrai des normes logiques? Comment pourrait-on ne pas accepter les normes de la logique, à moins d'être frappé d'irrationalité complète? Comme le disait Wittgenstein (ou c'est peut-être l'une des choses qu'il voulait dire par là) "on ne peut pas penser illogiquement". Vois-tu, les normes de la logique sont des normes universelles, des normes qu'on ne peut pas refuser sous peine de cesser simplement d'être membre de la communauté de ceux qui pensent?

- Soit, dit la Tortue, toujours conciliante. Admettons que les lois logiques concernent non pas ce que nous pensons actuellement, mais ce que nous pourrions penser si nous étions idéalement placés, comme agents idéalement rationnels. Je veux bien que ce soit une idée distincte de celle selon laquelle la logique décrit les lois de l' "être-vrai".

Laisse moi alors écrire sur mon calepin la proposition suivante: 
(F) Idéalement un agent rationnel qui accepterait que (A) et (B) sont vraies devrait accepter $(Z)$.

Mais même si l'on ajoute cette proposition, et même si je l'accepte, je ne vois toujours pas ce qui me conduirait à accepter (Z). Vois-tu, je ne suis pas, en tant que Tortue, un "agent idéalement rationnel". Je fais, comme nous autres animaux soi-disant rationnels, toutes sortes d'erreurs de raisonnement. Les psychologues sont très bien documentés là-dessus. Ils ont écrit toutes sortes d'études et mené toutes sortes d'expériences destinées à montrer que même dans le cas de raisonnements conditionnels très simples (comme la célèbre tâche de sélection de cartes de Wason), les agents s'écartent considérablement, dans leur comportement inférentiel effectif, des normes idéales de la logique, qu'il s'agisse de la logique déductive ou qu'il s'agisse des règles du calcul des probabilités d'ailleurs. Et étant donné que nous sommes prompts à faire toutes ces erreurs, pourquoi voudrais-tu que l'ajout, dans mon raisonnement, d'une prémisse telle que $(\mathrm{F})$ puisse mouvoir mon esprit à accepter ( $\mathrm{Z})$ ? Même si je reconnaissais, rationnellement, consciemment, avec réflexion, etc. que $(F)$ est vrai, et même si je décidais de m’y conformer parce que je me serais convaincue que tout agent rationnel doit accepter $(\mathrm{F})$ et agir en conséquence, je pourrais toujours, pour une raison quelconque, bonne ou mauvaise, refuser d'accepter (Z).

A ce point Achille entra vraiment dans une colère homérique.

— Mais le "doit logique" n'est-il pas aussi dur que le "doit" moral? Si je te dis que tu dois inférer la conclusion, n'est-ce pas exactement comme si je te disais que tu dois obéir à un impératif moral, par exemple que tu dois secourir les gens en détresse? Je comprends bien que quelqu'un qui ne reconnait pas cet impératif, ou qui refuse de le reconnaitre n'accomplisse pas l'action correspondante. Je comprends aussi que toutes sortes de raisons psychologiques, ou même de causes, 
pathologiques ou autres, puisse t'empêcher de te conformer à cet impératif. Mais à partir du moment où tu l'as reconnu et admis - ce que tu as fait - comment pourrais tu manquer te t'y conformer? Frege disait que les lois de l'être-vrai sont comme les lois de l'être-bon, et même celles de l'être-beau. Peirce, qui représentait plutôt la thèse de la rationalité idéale, soutenait que la logique, comme l'éthique et l'esthétique, sont des "sciences normatives", en un sens identique pour chacun des domaines, de la connaissance, de l'action, ou du jugement esthétique.

- Je suis très contente que tu aies toi-même fait ce rapprochement entre le cas du raisonnement logique et celui du raisonnement pratique, un rapprochement qu'Aristote lui-même faisait quand il parlait de "syllogisme pratique" pour parler des raisonnements qui nous conduisent à l'action à travers une délibération. Dans ces cas, le Stagirite soutenait que la conclusion du raisonnement n' est pas une proposition, mais une action, que l'agent effectue "euthus", immédiatement, à partir du moment où il voit la vérité d'une prémisse majeure qui lui présente une action comme bonne, ou désirable, ou en quelque sens valable, et d'une prémisse mineure, qui lui présente une certaine circonstance comme exemplifiant le type d'action désirable, bonne, ou valable. Mais je doute que ce rapprochement puisse nous aider à résoudre notre problème. Car on peut présenter, comme l'ont noté récemment Blackburn (1995) et Schueler (1995), pour le raisonnement pratique un paradoxe analogue à celui de Carroll pour le raisonnement logique. Considère le cas suivant, inspiré de celui présenté par Schueler.

Supposons que je m'apprête à m'installer dans le compartiment d'un train bondé, et qu'au moment où je pénètre dans le compartiment, une veille dame, chargée d'une grosse valise, s'apprête à en faire autant. Nous n'avons pas besoin de faire autre chose qu'échanger des regards pour que je comprenne que la situation comporte les données suivantes: 
(a) La vieille dame veut s'asseoir dans le compartiment où il ne reste qu'une seule place assise, et elle l'air déjà très fatiguée d'avoir porté sa valise sur le quai.

(b) Le train est bondé et il est plus que probable qu'il n'y a pas d'autre place assise, et que même s'il y en avait une dans un autre compartiment, elle n'aura pas le temps de s'y rendre avant qu'un autre voyageur la prenne.

(c) La veille dame est si fragile et timide qu'elle n'aura sûrement pas le courage de revendiquer dans un autre compartiment une place que pourrait lui céder un voyageur plus jeune qui pourrait se contenter de voyager dans le couloir.

(d) Je suis moi-même une tortue encore jeune et vaillante, malgré l'usure ferroviaire que je subis depuis près de vingt ans, et je fais suffisamment de train dans la semaine pour qu'un trajet dans le couloir ne fasse pas pour moi beaucoup de différence.

(e) Il me suffirait de lui céder ma place dans le compartiment pour satisfaire cette demande muette que je lis dans son regard las.

J'ai beau être Tortue, je porte suffisamment en moi la forme de l'humaine condition pour apprécier les faits (a)-(e). Et pourtant voilà que je m'apprête à prendre la place vide dans le compartiment. Mais voilà que le reagard de la vieille dame se fait plus insistant. Elle n'a pas même besoin d'ouvrir la bouche pour que je comprenne immédiatement qu'elle m’indique par là que les faits étayent fortement la conclusion suivante:

(f) C'est moralement mon devoir que de lui céder ma place dans le compartiment

Réalisant cela, je me fige, et mon pied — ma patte, veux-je dire- reste suspendu entre le couloir et le compartiment. C'est suffisant pour indiquer à la vieille dame que j'admets (f), et que j'apprécie toute la force morale que son injonction muette comporte. Et pourtant voilà que je m’apprête à nouveau à pénétrer dans le 
compartiment et à y prendre la place qui commence à m'attendre. Le regard insistant mais las de la vieille dame est toujours sur moi. Je comprends en une fraction de seconde qu'elle semble penser que la raison pour laquelle je ne lui cède pas la place est que j'ai peut-être une obligation morale plus forte encore que (f) - par exemple peut-être y a-t-il dans le compartiment un malade qui a besoin de mon assistance, et qui requiert ma présence durant le voyage. Un coup d'oeil rapide aux autres personnes présentes dans le compartiment lui révèle qu'elles sont étrangères à moi, et que personne ne m'y requiert. Je lis à présent dans son regard (je détaille les étapes, mais évidemment tout ceci se passe en des fractions infinitésimales de secondes) qu'elle entend me faire comprendre que, toutes choses égales par ailleurs, j'ai moralement le devoir de la laisser prendre ma place, autrement dit que:

(g) Comme il n'y a pas de raison morale pour que je ne lui cède pas ma place, je suis moralement tenue de la lui céder.

Et voilà que néanmoins je commence à pousser ma valise en direction de l'entrée du compartiment. Je saisis en un instant qu'elle peut croire qu'il y a pour moi une autre raison, non spécifiquement morale, de me joindre aux voyageurs (par exemple je m'apprête à prendre place auprès d'une très jolie Tortue qui ne semble pas farouche). Mais elle jette un regard rapide à mon alliance (les Tortues en portent aussi), et, supposant que je suis fidèle à mon épouse Tortue, elle comprend que le voisinage de la donzelle m'est indifférent. A nouveau je lis dans son regard las la proposition suivante:

(h) Comme je n'ai pas de raison de ne pas lui céder ma place, toutes choses égales par ailleurs je devrais la lui céder. 
Toujours conciliante, je lui jette un regard qui lui laisse entendre que j’admets cela aussi. Et pourtant, je pousse ma valise dans le filet, et m'assieds à la place libre dans le compartiment, laissant la vieile dame se débrouiller avec son énorme fardeau dans le couloir bourré de militaires goguenards en permission.

Si tu apprécies cette histoire, cher Achille, tu comprendras qu'elle est très similaire à celle de Lewis Carroll qui nous opposa jadis. Dans le cas du raisonnement logique, il faut distinguer trois choses (Schueler 1995,p.716):

(1) Les deux prémisses, (A) et (B) impliquent-elles logiquement (Z)?

Etant donné que je crois, pour de bonnes raisons, que (A) et (B) sont vraies, est-il raisonnable pour moi de croire $(Z)$ ?

(3) A supposer qu'il soit raisonnable pour moi de croire ( $Z$ ) sur la base de $(\mathrm{A})$ et $(\mathrm{B})$, qu'est-ce qui est supposé me conduire à croire $(\mathrm{Z})$ ?

La réponse à (1) est clairement: oui. Mais ce fait en lui-même ne permet pas de donner une réponse positive à (2). Tu peux me faire admettre un autre énoncé conditionnel (hypothétique, disions-nous) comme (C), ou (D) cela ne résoudra pas la question. Et tu peux aussi me dire que le modus ponens est une règle d'inférence valide de la logique, sanctionné par les théorèmes de complétude des règles de la logique propositionnelle, cela ne la résoudra pas non plus. Et finalement, même si j'ai de très bonnes raisons de croire $(Z)$ et de croire que la règle d'inférence en question est valide, il ne s'ensuit toujours pas que cela va me faire automatiquement croire que $(\mathrm{Z})$ est vrai.

Maintenant substitue dans ce qui précède "faire" à "croire", "avoir de bonnes raisons de faire" à "être raisonnable", et "croire que j'ai de bonnes raisons de faire" à "croire qu'il est raisonnable de croire", tu te trouveras face au même problème. Qu'il s'agisse du "doit" logique ou du "doit" moral, il n’y a rien dans ces énoncés normatifs des deux types qui rende nécessaire que nous croyions ou 
fassions les choses correspondantes. Croire le contraire, ce serait faire la même erreur que Socrate, quand il niait que la volonté puisse être faible, et soutenait que "Nul n'est méchant volontairement". Socrate soutenait cela parce qu'il pensait, comme l'on fait beaucoup de penseurs après lui, que dans le raisonnement pratique les prémisses nécessitent, logiquement (et déductivement) la conclusion. C'est pourquoi il n'admettait pas que l'on puisse voir le meilleur, et faire le pire. Pour lui l'individu akratique était nécessairement quelqu'un qui n'a pas vu correctement la vérité de l'un ou l'autre des prémisses du raisonnement pratique. S’il agit mal, c'est par ignorance, et non pas en connaissance de cause. Mais comme l'a soutenu Aristote, la faiblesse de la volonté, l'akrasia, est possible. Et comme l'a montré Davidson (1969), si elle est possible, ce n'est pas simplement au titre de l'expérience courante, mais aussi parce que le raisonnement en question est un raisonnement non pas déductif, mais inductif. La prémisse majeure du raisonnement n'est pas une proposition universelle catégorique. C'est plutôt une prémisse comparative de la forme "Toutes choses égales par ailleurs (et tout bien considéré, je juge qu'il vaut mieux faire A que B”. L'akratique juge cela, mais fait B. Il éprouve un conflit, mais son conflit n'est pas l'effet d'un contradiction formelle (déductive) entre sa conclusion (son action) et sa prémisse. Il en est de même, bien que l'on ne l'ait pas suffisamment aperçu, du raisonnement théorique, ou logique. Le fait que l'on tienne une prémisse comme raisonnable, et qu'on tienne qu'il est raisonnable de tenir comme raisonnable (ou que ce soit raisonnable pour un agent idéalement rationnel) de croire que cette prémisse implique une certaine conclusion, n'implique pas que l'on va croire cette conclusion. Tout comme il y a des akratiques en morale, il y a des akratiques en logique. Je confesse que je pourrais bien, en tant que Tortue, être un caractère de ce type.

— Je te concède, répondit Achille, la grande similarité des deux cas, au moins de prime abord. Mais je crois que je vois aussi à présent ce qui ne va pas dans ton raisonnement. Deux choses sont fallacieuses dans ce raisonnement, et chacune 
d'elles lui est fatale, que l'on soutienne ou non que le cas du raisonnement logique est le même que celui du raisonnement pratique.

Premièrement supposons que les deux cas soient similaires, et que ce qui t'arrête est l'impossibilité de passer d'un "doit" moral ou logique à un faire ou à un croire. Ton raisonnement repose en fait sur la thèse morale converse selon laquelle on ne peut pas passer de "est" à "doit" qui était celle de Hume. Mais ton propre exemple de la veille dame dans le train infirme cette thèse. Tu as décrit la scène de telle façon que, sur la base des faits (a)-(e) (et j'insiste bien sur le mot fait) tu éprouves l'obligation rapportée par (f). (f) est clairement un énoncé normatif ou déontique, alors que (a)-(e) sont des énoncés qui rapportent des matters of fact, comme dirait Hume. Mais en ajoutant la prémisse (f) sur la base des faits (a)-(e) tu as clairement toi-même commis l'erreur que Hume dénonçait: tu es passée d'énoncés contenait des "est" à un énoncé contenant un "doit". Peu importe que tu te contredises, si par ailleurs tu défends la coupure humienne entre être et devoir-être. Ce qui m'intéresse ici, c'est que des comptes rendus factuels puissent permettre de dériver une conclusion parfaitement normative, comme l'ont soutenu les critiques de Hume (cf. par exemple Searle 1969, Gardies 1987). Maintenant, puisque selon toi la situation est la même en ce qui concerne le raisonnement logique, pourquoi l'appréciation de faits logiques (que (Z) s'ensuit de (A) et de (B) logiquement) ne conduirait-elle pas, par une transition semblable, à l'appréciation du fait normatif que l'on doit inférer $(\mathrm{Z})$ ? Je veux dire qu'il y a une sorte de feedback ici: des faits nous conduisent à admettre des normes, lesquelles en retour justifient que les faits soient conformes à celles-ci.

— Me permets-tu, dit ici la Tortue, de répondre à cette objection avant d'entendre la seconde?

- Vas-y. 
- Je te ferai remarquer, premièrement, que mon énoncé (f), qui est clairement déontique, je te l'accorde, n'est pas le seul énoncé indiquant des raisons d'agir que j’ai pu invoquer. Il y a aussi (h) qui est un énoncé de motivation non déontique, invoquant une clause "toutes choses égales par ailleurs", qui le place clairement parmi ce que Kant appelle les impératifs hypothétiques. Donc la régression carrollienne peut s'enclencher dans ce cas même si je n'invoque aucune raison morale. Mais supposons que l'on invoque une telle raison, sous la forme (f) ou (g). En ce cas, même si j'ajoute (f) ou (g) à mon raisonnement, cela ne me conduira pas plus à faire l'action correspondante (ou à avoir la croyance correspondante) que (D), (D) etc. ne le faisaient dans le raisonnement de Carroll (Schueler 1995, p.720) . Donc même si je ne fais pas appel à la prémisse déontique en "doit", je ne suis toujours pas mue. Je suis un moteur non mû, comme aurait dit le Stagirite. Je suis en effet une Tortue humienne, et je pense que les transitions de "est" à "doit" ne sont pas légitimes. Mais c'est parfaitement indépendant du problème qui nous occupe. Quelles que soient les raisons, déontiques ou pas, que tu puisses m'apporter pour inférer ( $\mathrm{Z}$ ) de (A) et (B), je ne bougerai pas, que ce soit logiquement ou pratiquement.

- Admettons, répondit Achille. Mais alors laisse-moi te proposer ma seconde objection. Elle repose cette fois sur le rejet de ton assimilation entre le cas logique et le cas pratique. Ton assimilation des deux cas m'a en réalité fait voir ce qui ne va pas dans ton argumentation. Quand tu supposes que les deux cas sont similaires, logique et pratique, tu fais l'assimilation dans le sens inverse, du cas pratique au cas logique. Tu supposes en effet que le fait d'inférer ( $Z$ ) de (A) et (B) est une sorte d'acte. Et j'en veux pour preuve le terme que tu n'as pas cessé d'employer depuis le début de nos conversations, y compris dans celle qui nous opposa il y a un siècle: accepter une proposition. Selon la conception implicite que tu proposes, accepter une proposition est bien une forme d'acte, soit mental, soit linguistique, que plus traditionnellement les philosophes ont appelé "jugement", "assentiment", 
ou "Fürwahralten" (tenir-pour-vrai). Je peux reconnaitre, ou envisager une proposition, sans la tenir pour vraie, c'est-à-dire sans lui donner volontairement mon assentiment. Je peux aussi croire une proposition être fausse, et néanmoins l'affirmer, ou décider de la tenir pour vraie, au titre de ce que Kant appelait une croyance pragmatique (dans la Doctrine transcendantale de la méthode). Quand tu supposes que dans le raisonnement logique comme dans le raisonnement pratique, tirer la conclusion est quelque chose que tu fais, tu adoptes implicitement cette idée. Mais ce n’est pas ce qui se passe dans le cas logique. Dans le cas logique, il ne s'agit pas d'accepter une conclusion, ni encore moins d'accepter des prémisses auxiliaires, comme tu m'y incites. Il s'agit seulement de croire que la proposition est vraie, sans que la volonté intervienne. Ce que je me tue à te répéter depuis le début, c'est que ton esprit doit se mouvoir vers la conclusion, parce qu'il est, face à elle, passif, et entraîné par elle, exactement comme les logiciens disent qu'elle est "entrainée" par les prémisses. Il y a pire. En supposant que le raisonnement logique met en jeu des actes d'acceptation, et que la reconnaissance de la validité des règles logiques met en jeu aussi de telles actes, tu sosucris à une conception complétement "décisionniste" de la rationalité (Renaut-Mesure 1996). Tu supposes que les principes de la rationalité (logique ou pratique) sont l'objet d'un choix, qui pourrait être lui-même rationnel, et dont la rationalité de la rationalité serait elle-même affaire de choix, etc. Mais la rationalité d'une inférence n'est pas affaire de choix, pas plus que l'inférence elle-même. Elle s'impose à nous. Nous ne choisissons, ni n'acceptons rien. C'est ce qu'il y a de correct dans la conception fregéenne, même si sa mythologie platonicienne doit être rejetée.

- Je suis tout à fait d'accord, mon cher guerrier, pour faire la distinction que tu proposes entre croire et accepter. De bons auteurs (Cohen 1992, Engel 1994, 1995) l'ont avancée. Et je veux bien admettre en effet que j’ai un peu fait fond sur l'ambiguité entre les deux sens du mot "croyance", la croyance comme assentiment volontaire et comme assentiment passif. Mais quand j'ai dit que je 
refusais la conclusion $(\mathrm{Z})$ du raisonnement qui nous occupe, ce "refus" n'était pas volontaire au sens où tu le dis, c'est-à-dire au sens où le fait de tirer la conclusion d'une inférence serait une sorte d'acte mental intentionnel. Ou plus exactement: si j'ai refusé de tirer la conclusion c'est précisément parce que j'admettais d'entrée de jeu que je n'avais pas à la refuser ou à l'accepter! Si je n'ai pas accepté la conclusion, c'est tout simplement que je ne voyais pas de raison, bonne ou mauvaise, de l'accepter, même une fois que tu m'as donné ces raisons. On peut certes accepter quelque chose sans bonne raison, ou sans comprendre ce que l'on accepte, comme lorsqu'on croit ce que nous dit notre gourou, ou notre psychanalyste. On peut aussi décider de tenir une proposition pour vraie, juste parce que cela nous amuse. Mais la plupart du temps, nos actes d'acceptation sont fondés sur nos croyances, et sur les bonnes raisons, empiriques ou non, que nous avons de croire. Et ici mon point était simplement que même en ayant devant moi toutes les bonnes raisons du monde de croire la conclusion de l'inférence, et même de les accepter, je ne parvenais pas à la croire. Je suis parfaitement d'accord avec toi sur le fait que la croyance est passive, alors que certaines sortes d'assentiment ne le sont pas. Et je suis parfaitement d'accord avec toi pour reconnaitre que ce qui est en cause quand nous faisons une inférence, ce sont nos croyances, et pas des actes volontaires ou intentionnels d'une certaine sorte. Mon point était que même une fois que l'on me mettait sous les yeux toutes les bonnes raisons du monde, et les bonnes raisons de croire ce bonnes raisons justifiées, je ne parviendrais toujours pas à croire la conclusion sur la base de ces bonnes raisons.

La morale est la suivante. Croire, comme inférer, sont des états passifs de l'esprit. On croit pour des raisons, comme on agit pour des raisons. Mais quand on nous montre ces raisons comme des justifications de nos croyances ou de nos actions, ce ne sont pas ces justifications qui nous font croire ou agir. C'est quelque chose d'autre, quelque chose comme une force brute de nos esprits, du genre précisément de ce que Hume appelait “belief”. 
- Mais alors veux-tu dire que la seule explication possible du fait que nous suivons une règle de logique, ici celle du modus ponens, est une explication psychologique ? Veux-tu dire que la seule chose qui puisse expliquer le fait qu'on passe des prémisses à la conclusion est l'existence d'un processus mental, quelle que soit d'ailleurs la manière dont on l'analyse — qu'il s'agisse du traitement de représentations par l'esprit, de l'activation de règles d'inférences inscrites dans un "langage de la pensée" inné (Rips 1994), ou de la construction de "modèles mentaux" (Johnson-Laird 1991), ou quoi que ce soit d'autre? En ce cas, nous tombons de Charybde en Scylla: nous avons rejeté, et je me suis accordé avec toi finalement là-dessus, le platonisme frégéen selon lequel il suffirait d'avoir une intuition de lois platoniciennes pour reconnaittre la validité d'une inférence, mais voilà que nous retombons dans le psychologisme le plus plat. Tu sembles à noveau, comme tout à l'heure, confondre les raisons que nous avons d'inférer une proposition avec les causes (psychologiques ou autres) qui nous font l'inférer. Mais ce psychologisme n'est pas plus admissible que le platonisme des règles. Pas seulement en raison des arguments classiques que Frege et Husserl ont dirigés contre cette thèse. Il est aussi inadmissible pour des raisons que Wittgenstein a bien mises en valeur. A mon tour, à présent, de te poser quelques questions à ce sujet.

— Je suis prête.

(Ici Acbille prend une pose wittgensteinienne, il s'absorbe dans ses pensées, émet quelques borborigmes hésitants, se donne des airs profonds et mystérieux, et jette des regards furieux et sombres à son interlocutrice.)

- Hum! Bon! Euh! Mmm...Tu admets, n'est-ce pas, que quand je t’ai présenté les deux prémisses (A) et (B), et la conclusion (Z) qui s'ensuit, tu as compris ces prémisses et cette conclusion? 
- Oui, je les ai comprises.

— Tu admets, en particulier, que la règle d'inférence que tu as suivie est celle du modus ponens, "de A et de si A alors B, inférer B "? Et que tu comprends le sens de cette règle?

- Oui.

- Mais qu'est-ce qui te dit que tu as bien suivi cette règle? Qu'est-ce qui te dit que tu n'as pas en fait suivi une autre règle, comme "de A et de si A alors B, inférer $\mathrm{B}$ jusqu'à la millième fois, et inférer $\mathrm{C}$ à la mille et unième fois "?

Appelons cela la règle du schmodus ponens. Qu'est-ce qui te dis que tu n'as pas suivi la règle du schmodus ponens? Et ne serait-ce pas la raison pour laquelle tu n'as pas accepté la conclusion $(Z)$ que je te présentais?

— Je vois où tu veux en venir. Un sceptique quant à la signification peut me dire que je ne sais pas quelle règle je suis en réalité, et que je crois seulement suivre la règle $\mathrm{du}$ modus ponens, alors qu'en fait je pourrais en suivre une autre. Cela expliquerait que je n'accepte pas la conclusion, tout simplement parce que je n'ai pas compris la règle comme toi. Serais-tu toi même, Achille, un tel sceptique, au sens de Wittgenstein lu par Kripke (1981)?

- Non, je ne le suis pas, précisément, pas plus que Wittgenstein.

- Moi non plus. Je pense comme lui que quand nous suivons la règle nous la suivons "aveuglément" et que nous savons quelle règle nous suivons. J'ai invoqué la force brute du "belief' humien, mais mon point aurait pu être formulé aussi en ces termes wittgensteiniens. Mon point n'était pas de défendre une forme de scepticisme quant à la signification des mots, encore moins quant à la signification des mots logiques.

- Mais ton invocation du "belief", de la croyance, de la nécessité où nous sommes, même une fois qu'on nous a présenté explicitement la signification des mots "si...alors", "s'ensuit de", et de la règle du modus ponens semble impliquer que tu crois encore que la compréhension de ces mots et de cette règle est une afaire de psychologie, une affaire des processus et représentations mentales qui se 
déroulent en nous quand nous la comprenons. Or c'est précisément l'une des implications du "paradoxe" de Wittgenstein au sujet des règles: si nous supposons qu'il existe en nous un certain type de processus mental qui "encode" des règles d'inférences dans notre esprit, ou qui les "décode" au moment où nous comprenons, nous supposons aussi que "suivre une règle" est une affaire d'interprétation, de lecture par l'oeil de l'esprit ou par quelque processus inconscient, et par conséquent nous supposons qu'il existe une règle qui nous permet d'associer ces interprétations à la règle elle-même, et nous sommes embarqués dans la régression que dénonce, précisément, Wittgenstein. Or suivre une règle, nous dit-il justement, n'est pas affaire d'interprétation, pas une affaire de processus psychologiques conférant une signification mentale aux mots de notre langage. Ce ne sont pas des processus internes qui fixent notre usage des mots et notre activité de "suivre la règle".

- Je n'ai pas voulu le suggérer non plus, ou tout du moins je n'ai pas voulu suggérer que c'était tout ce qu'il y a en dire, même si je pense, contrairement à Wittgenstein, que nous ne devrions pas rejeter par principe ce genre d'explications. En fait la régression carrollienne a quelque chose de très proche de la régression que dénonce Wittgenstein. Wittgenstein nous dit que quand nous suivons une règle (ici une règle de logique) nous n'avons pas besoin de produire une autre règle pour interpréter la règle que nous suivons, sous peine de régression à l'infini. Carroll nous dit que quand nous suivons une règle, nous n'ajoutons pas cette règle comme une prémisse conditionnelle ou hypothétique à notre inférence, sous peine de régression à l'infini. Nous n'avons pas besoin, pour inférer la conclusion de considérer la proposition conditionnelle (C) comme vraie. Mais Carroll ne voulait pas dire non plus que la proposition conditionnelle $(\mathrm{C})$ est vraie nécessairement, plutôt que simplement vraie (de manière contingente). 3 Or c'était

\footnotetext{
3 Ici j'ai été aidé beaucoup par Railton (1996), qui développe le même thème en relation à la fois aux inférences logiques ou au raisonnement théorique et au raisonement pratique.
} 
bien, semble-t-il, la position que tu semblais adopter au départ, la position platonicienne.

- Comme je te l'ai dit, je n'accepte plus cette position. Je suis d'accord avec les logiciens qui font la distinction entre une proposition vraie et une règle d'inférence, et qui admettent qu'une règle d'inférence joue un rôle complètement différent.

- Alors tu noteras, comme nous l'avons fait plus haut, que la même chose vaut pour le raisonnement pratique: ce n'est pas parce qu'on nous présente une raison d'agir comme une prémisse hypothétique vraie, ou même comme une prémisse hypothétique nécessairement vraie (valant pour tout être rationnel en général) que nous sommes mus à agir. La notion même d'implication - tout comme la notion même d'action rationnelle_ présuppose que les règles de la logique — et les règles de l'action rationnelle- sont déjà là , que nous les maitrisons et que nous les appliquons. C'est pourquoi il est absurde de nous les présenter comme des propositions vraies que nous aurions à reconnaître avant d'inférer ou d'agir. Cela ne veut pas dire qu'elles sont là comme des propositions platoniciennes, que nous aurions à "saisir". C’est ce que je voulais dire quand j'invoquais la "force brute" du belief. Et je crois que sur ce point, je suis parfaitement d'accord avec Wittgenstein.

- Mais alors pourquoi parler de psychologie? Pourquoi ne pas admettre tout simplement, comme Wittgenstein, que suivre une règle est une pratique, qui n'est possible que parce que nous appartenons à une communauté qui reconnait ces règles, nous les impose, et s'accorde dans ses jugements sur celles-ci?

- Parce que je ne suis pas sûr que ce soit la réponse finale à la question de savoir pourquoi nous suivons les règles de la logique. Wittgenstein nous dit que suivre une règle est une activité, une pratique essentiellement sociale. Mais je ne veux pas exclure que ce soit, contrairement à ce qu'il soutient, une affaire de dispositions psychologiques. En d'autres termes, pour Wittgenstein la question: "La logique peut-elle mouvoir l'esprit?" est nécessairement mal posée si l'on invoque quelque chose comme l'esprit, ou le mental. Mais je crois quand même la question 
psychologique, formulée en ces termes, légitime. Pour moi croire est un état psychologique, naturel, qui ne laisse pas réduire à des déterminations sociales. Le naturalisme de Wittgenstein, sa conception de notre "histoire naturelle", est un naturalisme social. Mais il y a d'autres formes de naturalisme. Je ne veux pas exclure qu'un naturalisme psychologique, voire même biologique, soit la bonne réponse. Mais c'est une autre histoire ${ }^{4}$. Elle impliquerait que nous revenions en détail sur l'argument du langage privé, sur l'impossibilité d'une conception "internaliste" de la signification, et sur ce que cet argument est réellement supposé prouver.

- Je conviens que ce serait encore une autre affaire, et l'occasion peut-être de poursuivre encore notre dialogue. Contentons-nous, pour le moment, d'en rester là. La seule question qui me reste à te poser est celle-ci: cette conclusion, (Z), vastu ou non la tirer?

— Mais oui, bien sûr, je la tire: "Les deux côtés de ce triangle sont égaux entre eux." Je n'ai jamais dit le contraire! 5

\section{Références}

Blackburn, S. 1995 "Practical Tortoise Raising", Mind, 104, pp.696-711

Cohen, L.J. 1992An Essay on Belief and Acceptance, Oxford, Oxford University Press Dogson C.L. 1977, Lewis Carroll's Symbolic Logic, ed. W. Bartley II, New York, Clarkson

\footnotetext{
${ }^{4}$ Voir, pour plus de détails sur cette démarche, Engel (1996), Engel 1997

5 Une première version de cet article a été lue à l'Université de Provence en décembre 1996. Une seconde version, en anglais, a été présentée en mai 1997 au colloque "Modeli mentali" en l'honneur de P.N. Johnson Laird à l'Université de Padoue. Je remercie pour leurs remarques Eric Audureau, Ruth Byrne, François Clementz, Philippe Minh, Phil Johnson-Laird, Pierre Thibaud, ainsi qu'un expert anonyme pour Dialogue, et Peter Railton pour m'avoir communiqué son article (Railton 1996) où il traite de sujets voisins.
} 
Potter

Davidson, D. (1969) "How is Weakness of the will possible?", in Essays on Actions and

Events, Oxford, Oxford university Press 1980, tr.fr. Actions et événements, Paris, PUF 1993.

Dummett, M. 1973 Frege, Philosophy of Language, Duckworth, London Engel, P. 1989 La norme du vrai, philosophie de la logique, Paris, Gallimard, tr.angl. révisée

et augmentée The Norm of Truth, Harvester, Hemel Hamstead 1991 1994 "Believing, Accepting and Holding-True", inédit, Rapport du CREA

1995 "Les croyances", in D. Kambouchner, dir. Notions de philosophie, vol. 2,

Paris, Gallimard,p. 1-110.

1996 Philosophie et psychologie, Paris, Gallimard

1997 “Normes logiques et évolution”, Revue Internationale de Philosophie , 2 201-219

Geach, P.T. 1965 “Assertion”, Philosophical Review, repr. in Logic Matters, Blackwell, Oxford, 1972, pp.254-269

Johnson-Laird, P.N. \& Byrne, R. 1991 Deduction, Erlbaum, London \& Hillsdale Gardies, J.L. 1987, L'erreur de Hume, Paris, P.U.F

Granger, G. 1992 Formes, opérations, objets, Paris, Vrin

Kripke, S. 1981 Wittgenstein on Rules and Private Language, Blackwell, Oxford, tr.fr. T.Marchaisse, Règles et langage privé, Paris, Seuil 1996

Mc Geee 1985 “A Counterexample to modus ponens”, Journal of Philosophy, LXXXII,

9, 471-488

Railton, P.1996 ( à parâtre) "On the hypothetical and non-hypothetical in reasoning about 
belief and action"

Renaut, A. et Mesure, S. 1996 La guerre des dieux, Paris, Grasset

Rips, L. 1994 The Psychology of Proof, MIT Press, Cambridge Mass.

Ryle 1954 "If, So and Because” in G. Ryle, Dilemmas, Oxford, Oxford University Press, 1954

Searle, J. 1969 Speech Acts, Cambridge, Cambridge University Press, tr.fr. Les actes de langage, Paris, Herman, 1972

Smiley, T. 1995 “A Tale of Two Tortoises", Mind, 104, 725-736

Schueler G. 1995 "Why “Oughts" are not Facts", Mind 104, 712-723

Stroud, B. 1979 “Inference, Belief and Understanding”, Mind ,88, 179-195 